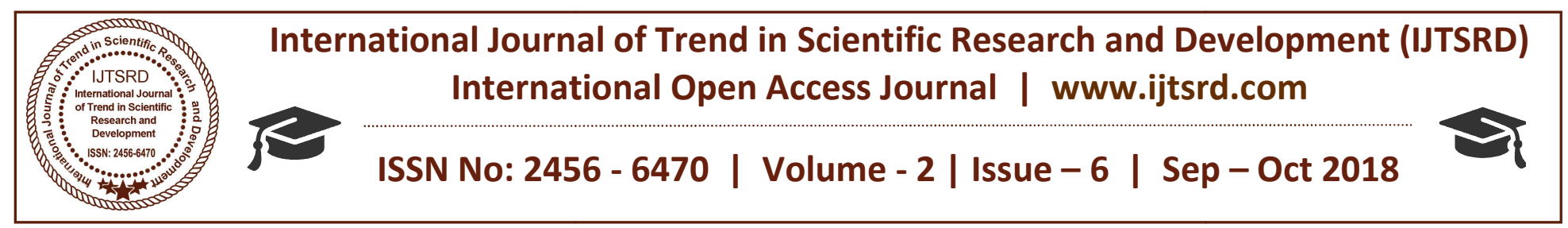

\title{
Role of Microbes Relating to Hygiene Hypothesis and Effect of Microbe Symbiosis on the Human Health- A Review
}

\author{
Rishabh Jain \\ Department of Zoology \\ Hansraj College, University of Delhi, New Delhi, India
}

\begin{abstract}
Since the microbes and humans have co-evolved with each other, so, the coexistence between them is key for survival on earth. With the emergence of concept that all microbes are germs in germ theory given by Robert Koch, the humans headed towards a lifestyle where hygienic environment became synonymous to the sterile environment. This eventually led to some unintended consequences. The millennial generation witnessed increased cases of allergic, atopic and autoimmune disorders. Interestingly, these cases are not uniformly distributed across the world. Rather, there is an uneven geographic distribution of these cases. The cases are more prevalent in developed/urbanised areas compared to underdeveloped/rural areas. The occurrence of these diseases is attributed to immune dysregulation occurring in the body. The uneven interactions between the microbes and the immune cells result in an exaggerated hypersensitive IgE mediated immune response which result in the diseases. The present review aims to discuss the role of microbes pertaining to hygiene hypothesis and the effect of microbe symbiosis on the human health.
\end{abstract}

Keyword: Microbes, Hygiene hypothesis, Microbiota, Diseases, Metabolites.

\section{INTRODUCTION}

The hygiene hypothesis proposed by David Strachan in 1989 aims to provide an explanation to immune dysregulation occurring in the allergic diseases [5]. It is based on Strachan's observation that hay fever is more common among the individuals with smaller household size i.e. there is an inverse relationship between the risk of hay fever and household size[5] [7]. The hypothesis in its original version suggests that decreased early life infections and low exposure to microbes alter immune system priming thereby increasing susceptibility to atopic diseases [7].

\section{Evolution of hygiene hypothesis}

With the advancement of technology and increased understanding of microbes, the hygiene hypothesis underwent more refinement and has evolved over time. Various extensions of the hygiene hypothesis have been given over time as shown in Fig. 1.

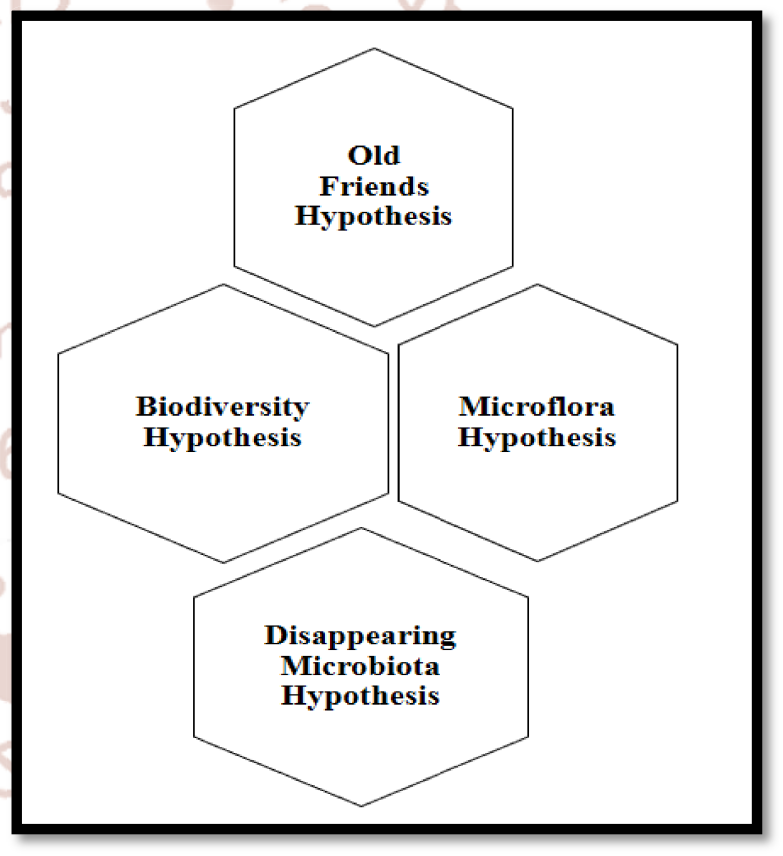

Fig. 1: Different Types of Hygiene Hypothesis

1. Old friends hypothesis: It was given by Rook et al. to more accurately describe Strachan's original hygiene hypothesis. It states that the organisms (macro and microorganisms) which coevolved with humans are necessary for the development of the immune system. Without these microbes, the immune system remain underdeveloped leading to atopic diseases [5]. 
2. Biodiversity hypothesis: This emphasizes that the greater diversity of microbes increases the immune tolerance and decreases the risk of allergic diseases [8].

3. Disappearing microbiota hypothesis: It suggests that reduced exposure to environmental microbes result in reduced immune tolerance and increased hypersensitivity disorders [8].

4. Microflora hypothesis: It is the modern day understanding of hygiene hypothesis. It emphasizes the importance of microflora composition in context to intestinal microbiota. The early life perturbations result in deviation from normal microbiota composition. This causes disruption of microbiota mediated mechanisms that confer immune tolerance. This eventually leads to hypersensitive disorders [5].

\section{Root cause of low exposure to environmental or human-associated microbes}

The western lifestyle and environmental pollution post industrialization are the main causes of lower microbe exposure.

1. Western lifestyle:It is one of the major reason for lower microbe exposure and consequently increased allergic diseases. So, hygiene hypothesis is often called as Western Lifestyle Hypothesis [7, $8,11]$. Some of the key factors of lifestyle that influence microbe exposure are:

$>$ Antibiotic consumption: The antibiotic treatment severely reduces microbiota composition and thus, lead to low exposure of microbes to the host's immune system [11].

> Improved health care standards: With the improved healthcare standards, the rate of infectious diseases has decreased. Moreover, people have gone so obsessed with cleanliness such that they try to maintain a sterile environment. Thus, people are less exposed to microbes such that their immune system remains underdeveloped and they are becoming more prone to allergic diseases [6].

$>$ Smoking: Smoking disrupts the microbial composition of mouth, airway as well as of the surrounding environment. This affects the immune system interactions of a host with microbes such that these altered interactions make host more prone to allergic diseases [2, 4].

$>$ Preferring Caesarean birth over natural birth: In natural birth, the infant is exposed to vaginal and faecal microbiota of mother and thus is exposed to diverse microbes which sensitise its immune system. In caesarean births on other hands, an infant is exposed to microbes of the hospital environment and those on the skin of workers. This leads to colonization by pathogenic bacteria and leads to microbial symbiosis [10].

2. Environmental conditions

post industrialisation:Increased pollution in environment alters the composition of environmental microbes and results in low exposure of microbes living in that region such that they become more susceptible to allergic diseases [7-8].

Role of microbes and their metabolites in hygiene hypothesis

Microbes and their metabolites have utmost important roles in human health and thus in the hygiene hypothesis. The lower microbe exposure in urban areas is suggested to be the major cause for a higher prevalence of atopic diseases in those areas [10] [13]. At farms, people are exposed to higher levels of bedding endotoxins (cell wall component of gram negative bacteria) and diverse microbial compounds from an early age. These endotoxins and microbial compounds act as an antigen, stimulate the immune system of the host and thus induce immune tolerance. So, at farms, people less susceptible to allergic diseases. We infer that there is an inverse relation between microbe exposure and atopic diseases [6] [8] [10]. These microbes thus have 2 roles to play [13] prevent colonisation of pathogenic bacteria in airways and interact with the innate immune system of a host, sensitize it and help in its development. This is further supported by the in yitro cytokine production which is inversely proportional to microbe exposure [13]. Moreover, mice grown in a germ free environment show decreased immune tolerance and lead to hypersensitive responses [11].Thus, considering the role they play and above evidences, we conclude that exposure to environmental microbes is necessary to prevent atopic diseases.

These metabolites produced by bacteria play a protective role by offering protection against allergic diseases. The role of these metabolites is below [22]:

1. Long chain fatty acids (LCFA):The human microbiota is involved in the metabolism of LCFA. They produce $\omega-6$ - fatty acid derived metabolites. Hydroxyl fatty acids such as 10hydorxy-cis-12-octadenoic acid derived from $\omega-6$ 
fatty acids are involved in maintaining tight junctions and epithelial barrier via GPR-40. If there is increased production of IL-4 producing helper T cells type 2 (Th-2) and decreased T-regs in atopic diseases.

2. Short chain fatty acids: Evidence suggests that SCFAs prevent immune dysregulation since they have anti-inflammatory property. They induce dendritic cells to achieve increased phagocytic capacity and reduce the ability to promote effector functions of Th-2 cells. They help in T-reg cell induction and help in barrier function in the intestine.

3. Bacterial amino acids: The several lines of evidence suggests the importance of these amino acids in conferring protection against allergic diseases. Melatonin (a metabolite of Dtryptophan), dietary histidine prevent the production of an inflammatory cytokine. Glutamine is necessary for induction of IL-10 producing intraepithelial lymphocyte in the small intestine. Glutamate produces immune tolerance. D-tryptophan increases t-reg cells and increasesTh-2 response in gut and lungs.

\section{Human Microbiota}

The microbes residing in or on the human body are collectively termed as human microbiota [1][8]. The human associated microbes form a super organism called holobiont [2]. The importance of human microbiota was emphasised in microflora hypothesis which suggests that alteration in human microbiota composition result in atopic diseases. The overall metabolic and immunological functions associated with human microbiota are described below $[1,14]$ :

$>$ Differentiation and maturation of mucosa and immune system of a host

$>$ Processing and detoxification of environmental chemicals

$>$ Maintenance of skin and mucosal barrier function

$>$ Development and regulation of the immune system and fine tuning of its reaction pattern i.e. the balance between pro and anti-inflammatory process.

$>$ Show colonisation resistance/bacterial interference i.e. prevent invasion and growth of disease promoting microorganisms

$>$ Metabolism regulation and control of fat storage

$>$ Food digestion and nutrition

\section{Types of Human Microbiota}

1. Skin microbiota: The skin microbiota in their conventional composition prevent invasion by pathogenic bacteria. For e.g.: Staphylococcus epidermis inhibits the growth of Staphylococcus aureus by the production of serine protease called Esp. Propionibacterium acnes has the ability to inhibit MRSA. These microbes strengthen host's immune system by influencing the functionality of host immune cells. Staphylococcus epidermis boosts immunity through increased expression of peptides like $\beta$-defensins. These microbes act as epidermal barriers [16][17]. The perturbations such as washing of hands, use of cosmetics etc. alter microbiota composition by removing the commensal microbes and providing ground for thereby reducing host's immune tolerance by influencing microbe-host immune system interactions.

2. Oral microbiota:Prevent the growth of exogenous species through the production of antimicrobial compounds [18]. For e.g.: Streptococcus viridans prevent colonisation by Streptococcus pneumonia by production of antimicrobial compounds [2] [4] and they interact with immune cells and confer immune tolerance. Perturbations alter the oral microbiota composition resulting in disruption of interactions between an immune system and oral microbiota. The lower exposure to microbes alters the composition and thus, reduces oral tolerance[2,4]. E.g. the high degree of biofilm aggregation promote periodontist in individuals susceptible to the disease leading to a nonresolving inflammatory response in connective tissue [2].

3. Nasopharynx and lung microbiota: It is useful to modulate local inflammatory responses, prevent colonisation by pathogenic bacteria and sensitise immune system[19] [20]. Alteration in microbiota due to pollution and lifestyle disrupts the microbiota-immune system interactions thereby decreasing immune tolerance of the host. The microbial overburden and overall decreased diversity of microbiota are observed [8]. For e.g.: asthma occurs due to increased Actinobacter and Klebsiella species in the lungs. Moreover, the germ free mice develop allergic diseases but on adding conventional microbiota reduced inflammatory responses are observed [7].

4. Gut microbiota: The gut microbiota plays an important role in the following ways i.e. compete for attachment sites in a border of epithelial cells 
and prevent colonisation by pathogenic bacteria [21], they interact with the innate immune system and induce regulatory $\mathrm{T}$ cells( T-reg cells) [7] and help in $\mathrm{T}$ cell priming and sensitisation of host immune system [7]. Alterations in human gut microbiota can have some serious implications on human health. The decrease in beneficial microbes give an opportunity to opportunistic pathogenic bacteria to invade the host and cause allergic diseases. For e.g.: increased Clostridium difficulty is linked to asthma [7]. Fermicutesin their normal composition produce short chain fatty acids (SCFAs) which have an anti-inflammatory effect but in increased number, it can lead to IBS (irritational bowel syndrome)[21]. Higher Enterobactereaceae: Bacteroidetes ratio lead to food allergies [8].

\section{Mechanisms associated with microbes in hygiene hypothesis:}

Microbes follow different mechanisms to confer protection against allergic and atopic diseases:

1. Th-1 and Th-2 deviation: Th-1 cells confer cell mediated immunity i.e. they activate macrophages which kill microbes through Reactive Oxygen Species (ROS). Th- 2 cells on other hand produce cytokine to activate alternative macrophage pathway to inhibit classical macrophage pathway and contribute to $\operatorname{IgE}$ and allergic responses[6][23]. Thus a balance between $\mathrm{Th}-1$ and Th- 2 cells is necessary. This balance is induced by exposure to conventional microbiota composition.In the developed countries, due to lower microbe exposure, there is a shift from Th-1 immunity to Th-2 phenotype. Further, Th-2 cells secrete cytokines which inhibit Th-1 pathway and causes an overburden of Th- 2 cells leading to allergic diseases.

2. Immunoregulation: T-reg cells secretion is induced by infections i.e. exposure to microbes. The role of T-reg cells is to suppress Tcell activity.CD4+CD25+ suppress Th-2 response leading to decreased atopic responses in individuals exposed to allergens like pollens. Moreover, the individuals suffering from atopic disorders, reduced a CD4+CD25+ expression is observed.

3. Antigenic competition/homeostasis: It means that 2 immune responses elucidated by distinct antigens occurring simultaneously tend to inhibit each other[6]. The development of strong immune responses against an antigen from infectious agent inhibit responses to a weak antigen like autoantigens and allergens. Lymphocyte competition for cytokines, recognition of $\mathrm{MHC}$ (major histocompatibility complex)/ self-peptide complex and growth factors are necessary to differentiation and proliferation of $\mathrm{B}$ and $\mathrm{T}$ cells during immune response within the frame of lymphocyte homeostasis.

4. Non antigenic ligand: Numerous experiments indicate that infectious agents promote protection against allergic diseases through mechanisms that lead to stimulation of non-antigenic specific receptors. For eg: Toll Like Receptors(TLR) stimulate could prevent the onset of spontaneous autoimmune diseases such as T1D in NOD mice, an observation made for TLR-2,-3,-4,-7 and -9 . Treatment with TLR agonists before disease onset prevents disease progression completely.

5. Gene-environment interactions: One of the interesting approaches to understand mechanisms associated with hygiene hypothesis consist of looking for associations between allergic diseases and polymorphisms of various genes especially the genes involved in the production of molecules involved in immune responses. For eg: the polymorphism in genes of innate immune responses such as CD14, TLR2, TLR4, TLR6 or TLR10 and intracellular receptors such as NOD1 (nucleotide binding oligomerization domain containing protein 1) and NOD2 (nucleotide binding oligomerization domain containing protein 2). CD14-159CT polymorphism and allergic inflammation.

\section{CONCLUSION:}

Thus we conclude that the microbes play a significantly important in the human health. A slight alteration in the composition of the microbes or less exposure to the microbes often results in allergic and atopic diseases. These microbes moreover can be used as probiotics to improve the health of individuals and prevent the occurrence of the atopic diseases. We also infer that the western lifestyle and thus, the environmental conditions are the root cause of the increased atopic diseases. We agree that cleanliness is important but beyond a certain extent, it is a problem for us since the maintenance of sterile environment results in increased cases of allergic and atopic diseases. 
International Journal of Trend in Scientific Research and Development (IJTSRD) ISSN: 2456-6470

\section{REFERENCES:}

1. Sultan, A. S., Kong, E. F., Rizk, A. M., \& JabraRizk, M. A. (2018). The oral microbiome: A Lesson in coexistence. PLoS pathogens, 14(1), e1006719.

2. Kilian, M., Chapple, I. L. C., Hannig, M., Marsh, P. D., Meuric, V., Pedersen, A. M. L., ... \&Zaura, E. (2016). The oral microbiome-an update for oral healthcare professionals. British Dental Journal, $221(10), 657$.

3. Vandegrift, R., Bateman, A. C., Siemens, K. N., Nguyen, M., Wilson, H. E., Green, J. L., ... \& Hickey, R. J. (2017). Cleanliness in context: reconciling hygiene with a modern microbial perspective. Microbiome, 5(1), 76.

4. Arbes Jr, S. J., \& Matsui, E. C. (2011). Can oral pathogens influence allergic disease?. Journal of Allergy and Clinical immunology, 127(5), 11191127.

5. Stiemsma, L. T., Reynolds, L. A., Turvey, S. E., \& Finlay, B. B. (2015). The hygiene hypothesis: current perspectives and future therapies. ImmunoTargets and therapy, 4, 143.

6. Okada, H., Kuhn, C., Feillet, H., \& Bach, J. F. (2010). The 'hygiene hypothesis' for autoimmune and allergic diseases: an update. Clinical \& Experimental Immunology, 160(1), 1-9.

7. Stiemsma, L. T., \&Turvey, S. E. (2017). Asthma and the microbiome: defining the critical window in early life. Allergy, Asthma \& Clinical Immunology, 13(1), 3.

8. Riiser, A. (2015). The human microbiome, asthma, and allergy. Allergy, Asthma \& Clinical Immunology, 11(1), 35.

9. Brookes, L., \& Cheng, L. E. (2015, april 6). Medscape. Retrieved from Medscape Medical Students.

10. Beigelman, A., Weinstock, G. M., \& Bacharier, L. B. (2014, april). The relationships between environmental bacterial exposure, Current opinion in allergy and clinical immunology, 14(2).

11. Penders, J., Gerhold, K., Thijs, C., Zimmermann, K., Wahn, U., Lau, S., \&Hamelmann, E. (2014). New insights into the hygiene hypothesis in allergic diseases: mediation of sibling and birth mode effects by the gut microbiota. Gut microbes, 5(2), 239-244.

12. Bull, M. J., \& Plummer, N. T. (2014). Part 1: The human gut microbiome in health and disease.
Integrative Medicine: A Clinician's Journal, 13(6), 17.

13. Dickson, R. P., Erb-Downward, J. R., \&Huffnagle, G. B. (2013). The role of the bacterial microbiome in lung disease. Expert review of respiratory medicine, 7(3), 245-257.

14. Fouda, E. M. ( 2017, May). Airway Microbiota and Allergic Diseases: Clinical Implications. International Journal of Pulmonary \& Respiratory Sciences, 1(5), 1-5.

15. Hospodsky, D., Pickering, A. J., Julian, T. R., Miller, D., Gorthala, S., Boehm, A. B., \&Peccia, J. (2014). Hand bacterial communities vary across two different human populations. Microbiology, 160(6), 1144-1152.

16. Sanford, J. A., \& Gallo, R. L. (2013, November). Functions of the skin microbiota in health and disease. In Seminars in immunology (Vol. 25, No. 5, pp. 370-377). Academic Press.

17. Grice, E. A., \& Segre, J. A. (2011). The skin microbiome. Nature Reviews Microbiology, 9(4), 244.

18. BOTERO, L. E., Delgado-Serrano, L., Cepeda Hernandez, M. L., Del Portillo Obando, P., \& Zambrano Eder, M. M. (2016). The human microbiota: the role of microbial communities in health and disease. ActaBiológicaColombiana, 21(1), 5-15.

19. Gao, Z., Kang, Y., Yu, J., \& Ren, L. (2014). Human pharyngeal microbiome may play a protective role in respiratory tract infections. Genomics, proteomics \& bioinformatics, 12(3), 144-150.

20. Dickson, R. P., Erb-Downward, J. R., \&Huffnagle, G. B. (2013). The role of the bacterial microbiome in lung disease. Expert review of respiratory medicine, 7(3), 245-257.

21. Bull, M. J., \& Plummer, N. T. (2014). Part 1: The human gut microbiome in health and disease. Integrative Medicine: A Clinician's Journal, 13(6), 17.

22. Hirata, S. I., \&Kunisawa, J. (2017). Gut microbiome, metabolome, and allergic diseases. Allergology International, 66(4), 523-528.

23. Tropper, Y. (2015). Understanding the Hygiene Hypothesis and its Mechanisms. The Science Journal of the Lander College of Arts and Sciences, 8(2), 10. 